\title{
Role of Bone Marrow Biopsy for Staging New Patients with Ewing Sarcoma: A Systematic Review
}

\author{
Kevin Campbell ${ }^{1}$, David Shulman ${ }^{2}$, Holcombe Grier ${ }^{3}$, and Steven DuBois ${ }^{4}$ \\ ${ }^{1}$ Children's Hospital Boston \\ ${ }^{2}$ Boston Children's Hospital \\ ${ }^{3}$ Dana Farber Cancer Institute \\ ${ }^{4}$ Dana-Farber Cancer Institute
}

September 21, 2020

\begin{abstract}
The incidence of bone marrow metastasis (BMM) in newly diagnosed Ewing sarcoma (ES) is variable across studies. An optimal staging strategy for detecting BMM is not defined. While BM biopsy and/or aspirate (BMBA) have been the gold standard, [F-18]fluorodeoxyglucose positron emission tomography (FDG-PET) to detect BM metastasis may decrease reliance on BMBA. We conducted a systematic review to assess incidence of BMM and the role of FDG-PET. We observed a pooled incidence by BMBA of BMM of $4.8 \%$ in all newly diagnosed ES patients and $17.5 \%$ among patients with metastatic disease. Only $1.2 \%$ of patients had BMM as their sole metastatic site. FDG-PET detection of BMM compared to BMBA demonstrated pooled $100 \%$ sensitivity and $96 \%$ specificity, positive predictive value of $75 \%$ and negative predictive value of $100 \%$. In the era of FDG-PET imaging, omission of BMBA may be considered in patients with otherwise localized disease after initial staging studies.
\end{abstract}

\section{Introduction}

Ewing sarcoma (ES) is an aggressive cancer of the bone and/or soft tissue and has peak incidence in the adolescent and young adult population. Behind osteosarcoma, it is the second most common bone cancer found in children, comprising 10-15\% of all primary malignancies of the bone.[1] At initial diagnosis, metastatic status is the most important prognostic factor, with survival rates diverging for patients with localized disease compared to those presenting with metastatic disease.[2] Common sites of metastasis include the lungs and bone, with bone marrow (BM) metastasis less commonly reported. Among patients with metastatic disease, those with lung metastases alone have a better prognosis, with event-free survival (EFS) ranging from 29-52\% while patients with bone/BM metastases have an EFS of $20 \%$ or lower.[2,3] Metastatic BM involvement has further been reported as an independent risk factor distinct from multiple bone metastasis. $[2,4,5]$ In one large analysis, patients with both $\mathrm{BM}$ and bone metastasis had inferior 3-year OS and EFS (21\% and 14\%) compared to patients with bone metastasis but no BM metastasis (37\% and 31\%).[2] Accurate identification of metastatic pattern is important in determining treatment, monitoring response, and determining prognosis.

Staging usually includes imaging of the primary site plus evaluation of metastatic disease with a chest CT (computed tomography), [F-18]fluorodeoxyglucose positron emission tomography (FDG-PET) scan, and bone marrow biopsy and aspirate (BMBA). The current gold standard evaluation for detection of metastatic BM disease has been BMBA. For example, the Children's Oncology Group required this procedure for enrollment on the last two protocols for patients with newly diagnosed disease. In adult patients with primary bone cancer, a BMBA is not routinely performed, and the current National Comprehensive Cancer Networks (NCCN) guidelines recommend an MRI of the spine and pelvis as an alternative to BMBA. [6] A 
BMBA procedure is invasive and carries a small risk of complications, $[7]$ including risks of general anesthesia administration in young patients. The increased use of FDG-PET scan, a non-invasive technique allowing for whole-body staging, has prompted investigators to ask if this modality may replace BMBA for detection of BM metastasis, [8-12] with several cohort studies directly comparing FDG-PET to BMBA in detection of BM metastasis. [13-18] Other studies have evaluated the use of FDG-PET/CT scan in ES and rhabdomyosarcoma and demonstrated FDG-PET scan to have a high sensitivity and specificity for detection of bone metastases when compared to bone scan and MRI.[17,19-22] FDG-PET scans have been shown to be superior to bone scans to detect asymptomatic metastasis in ES patients.[15]

To provide clarity on the incidence of metastatic BM disease in ES and the best strategy to diagnose BM disease at presentation in these patients, we pooled available evidence in this systematic review. First, we describe the incidence of metastatic BM disease with and without the presence of additional metastatic disease in the presentation of newly diagnosed ES as well as clinical associations with BM metastatic disease. Second, we report the available sensitivity, specificity, positive predictive value (PPV) and negative predictive value (NPV) of FDG-PET scans for diagnosis of metastatic BM disease in newly diagnosed ES patients.

\section{Methods}

This review was conducted according to the Preferred Reporting Items for Systematic Reviews and Metaanalyses (PRISMA) guidelines [16]. The review protocol for this study was prospectively registered at PROSPERO (registration number 179382).

\subsection{Search strategy}

Searches were conducted in April 2020 in three publicly available databases: PubMed; Google Scholar; and Cochrane Library. Search strategy involved an iterative process in which keywords for searches were a combination of one diagnosis name and one topic name. The Ewing sarcoma family tumors (ESFT) historically included a family of morphologically similar malignancies but with diverging names. Thus, five diagnosis search terms were utilized: Ewing sarcoma; Ewing's sarcoma; Peripheral Neuroectodermal Tumor; PNET; and Askin Tumor. Alongside a diagnosis term, 13 topic search terms were utilized: bone marrow; bone marrow biopsy; bone marrow aspirate; Positron Emission Tomography; PET; metastatic; metastatic pattern; metastasis; randomized; phase III; clinical trial; staging; and diagnosis. This process resulted in 65 individual searches ( 5 diagnosis terms x 13 topic terms) for each of the 3 databases utilized (195 total searches across all databases). For each search, a single reviewer documented the number of results, the number of abstracts read and the number of articles meeting criteria to be shared with a second reviewer for final approval. A third reviewer was available to resolve any discrepancies. Searches resulting in $>500$ results were edited to restrict specific keywords deemed irrelevant to this study. For example, the PubMed search of 'Primitive Neuroectodermal Tumor' + 'metastatic' yielded 4,129 results. After designating the search not return articles with keywords 'case report,' 'neuroblastoma,' 'medulloblastoma,' and 'pancreatic,' results decreased to 284 articles. To expand our search, references of the retrieved articles were also screened for additional studies.

\subsection{Eligibility criteria}

Clinical trials (phase I, II and III), prospective and retrospective cohort studies, cross-sectional studies, and case series of patients with ES were all considered for inclusion in this review. Case reports, reviews, viewpoints or conference reports were excluded. Studies in non-English language were excluded. Results from 1970-2020 were included, though studies incorporating data both after and before 1970 were excluded if it was unclear which data/patients met inclusion criteria for dates. If data were identified to be reported in $>1$ study, the study with the higher 'n' was selected.

\section{Data extraction}

The data collected for each study in the systematic review included: authors; journal; year of publication; country of origin; study design; study population; and study sample size. For references providing only 
aggregate data, the following were collected: percentage of patients with BM metastasis; percentage of patients with isolated BM metastasis; clinical predictors of BM metastasis; sensitivity of FDG-PET scans to detect BM metastasis; specificity of FDG-PET scans to exclude BM metastasis; PPV of positive FDGPET scans for "true" BM metastasis by biopsy; NPV of negative FDG-PET scans for no BM metastasis by biopsy; and any other imaging features associated with BM metastasis. For references providing patient level data, the following was also collected when available: age of each ES patient; primary tumor site of each ES patient; BM metastasis status (if present, subcategorized as either isolated BM metastasis vs BM + additional areas of metastasis); results of FDG-PET scan if completed at diagnosis; and results of unilateral or bilateral BMBA at diagnosis (if yes, was BM disease identified).

\subsection{Data synthesis}

To determine the incidence of BM metastasis in newly diagnosed ES patients, studies reporting newly diagnosed BM metastatic disease in a homogenous background population of all newly diagnosed ES patients were included. Studies reporting the incidence of BM metastatic disease in patients with metastatic disease were used for a separate calculation. The number of patients from each study was totaled to provide each patient with uniform weight in calculations of descriptive statistics.

To determine the sensitivity, specificity, PPV and NPV of FDG-PET scans in comparison to BMBA for the presence of BM metastasis in newly diagnosed ES patients, studies were included if results of FDG-PET scans and BMBA for diagnosis of BM metastasis were provided. A study was considered to sufficiently report on the status of BM metastasis via FDG-PET scan if it reported FDG-PET BM results separately from primary tumor or metastatic bone lesions. Studies were excluded if they reported the incidence of bone metastasis but not BM metastasis by FDG-PET scans.

\section{Results}

\subsection{Study selection}

The initial search identified 11,110 titles reviewed (PubMed: 8,324; Google Scholar: 1,208; Cochrane library: 1,578). Reviewer 1 read 330 abstracts, from which 97 articles (including 17 articles screened from references) were flagged for Reviewer 2 (Fig. 1). Seventy-five articles were identified for full-text review. Of these, 37 were deemed ineligible for inclusion due to the following reasons: 18 did not report incidence of BM metastasis; 7 did not utilize FDG-PET scans to assess for BM metastasis; 6 had missing data on diagnostic testing used for metastatic disease; 4 were not in English; 2 involved patients with diseases other than ES.

\subsection{Quantitative analysis: Incidence of BM metastasis}

We identified 10 studies providing quantitative data describing the incidence of BM metastasis in all unselected patients with newly diagnosed ES (Table 1).[21,23-31] These studies ranged in year of publication from 1995 - 2020. Eight studies were completed in a retrospective manner, one was a phase 3 clinical trial and one was a pilot trial. Combined, they comprise 1,663 patients (median 75 patients/study; range 17 647 patients).

Of the total of 1,663 patients, 79 had BM metastasis, for an incidence of $4.8 \%$ in this unselected group of patients. Among the 450 patients with any metastatic disease, the incidence of BM disease was $17.5 \%$ (79/450). Three studies reported the presence or absence of isolated BM metastases, with 1.2\% (8/692) of patients with isolated BM metastasis. $[24,25,27]$ Ten additional patients with isolated BM metastatic disease were identified in the literature for a total of 18. Eight of these 18 cases reported the location of the primary tumor: three pelvic primary tumors and five located in the appendicular skeleton.

We identified an additional 8 studies for which inclusion was restricted to newly diagnosed ES patients with metastatic disease only or a specific subset of metastatic ES. Five of these studies reported the incidence of BM metastasis in a selected population of ES patients (Table 2).[2,32-35] Of these, three excluded patients with newly diagnosed localized ES and reported on 934 total patients with newly diagnosed metastatic ES. Of these, $19.0 \%$ were reported to have BM metastasis. Two other studies describe further subsets of 
metastatic ES patients. One excluded patients with lung only metastatic disease and reported an incidence of BM metastasis of $43.8 \%$ (123/281).[2] A second study limited its inclusion to patients with metastatic bone ES and reported an incidence of BM metastasis of $23.7 \%$ (23/97).[35]

Two additional studies in patients with metastatic disease reported patients with isolated BM metastatic disease without providing an incidence of any BM metastasis. One study reported $7 \%(8 / 110)$ of patients with newly diagnosed metastatic ES to present with isolated BM metastasis, [36] another reported 3.3\% (2/60) incidence of isolated BM metastatic disease.[37]

Patients with isolated lung metastasis have superior outcomes compared to patients with other metastasis.[3] Since detecting BM metastasis in patients who might otherwise have a more favorable outcome may provide important prognostic information, we also assessed the frequency of BM metastases in patients with lung metastasis but lacking bone metastases. We evaluated all studies included in Tables 12 . Six of the 15 studies analyzed reported relevant data, with $8.8 \%$ (20/225) of patients with reported lung metastatic disease also having BM metastasis but not bone metastasis.

\subsection{Quantitative analysis: FDG-PET scan vs. BMBA}

Four studies (Table 3) were identified which utilized FDG-PET scans for staging of BM metastasis in newly diagnosed ES patients. $[21,29,38,39]$ Data from these studies were aggregated for quantitative analysis to define testing characteristics of FDG-PET scans compared to BMBA. The studies ranged in publication year from 2013 - 2019 and all were completed in a retrospective manner. Combined, these studies comprised 142 patients (median 23 patients/study; range 16-80 patients).

Pooled patient level data were used to construct a contingency table to determine sensitivity, specificity, PPV and NPV. Results revealed FDG-PET scans to have a sensitivity of 100\% (3/3) and specificity of $96 \%$ (96/100) to detect BM metastasis. Compared to BM biopsy, the PPV of a positive FDG-PET scan was found to be $75 \%(3 / 4)$ and the NPV of a negative FDG-PET scan was 100\% (106/106). The patient with a negative BMBA but positive FDG-PET scan was identified by Kasalek et al on FDG-PET scan to have BM metastatic lesions involving bilateral posterior iliac crest and extensive BM involvement elsewhere in the axial and appendicular skeleton. [38]

\subsection{Qualitative analysis}

Several studies were selected for inclusion in our review based upon qualitative analysis or descriptive findings contributing to the understanding of the incidence of BM disease at presentation in ES as well as the advantages and disadvantages to BMBA and FDG-PET scan in assessment of BM metastatic disease.

The most common reported association for patients with BM metastasis was the presence of other metastatic lesions, and specifically bone metastasis. Kopp et al found BMBA to be positive in $41.9 \%$ (13/31) of patients with the presence of other metastatic but $0 \%(0 / 85)$ in ES patients with non-metastatic disease.[26] A positive association of the number of bone metastatic lesions and presence of BM metastasis was reported by Inagaki et al. Of 26 patients with newly diagnosed ES, $100 \%(3 / 3)$ of patients with BM metastasis had bone metastases and $4.3 \%(1 / 23)$ patients without BM metastases had bone metastases.[29] Newman et al reported 0 of $91 \mathrm{ES}$ patients with $\mathrm{BM}$ metastases in cases without osseous metastatic disease.[21] Khan et al reported that $45.5 \%$ (5/11) of patients with BM mets had concurrent bone metastatic disease.[25]

Additionally, BM metastasis was reported to be associated with primary tumors of the pelvis, large tumor size, and presentation with fever and/or elevated lactate dehydrogenase (LDH) level. Two studies reported that pelvic primary site was significantly associated with BM metastatic disease at diagnosis. One reported a risk of $\mathrm{BM}$ involvement three times higher in patients with pelvic lesions compared with other primary sites; the second reported BM involvement in $22 \%$ (13/59) of patients with pelvic primary disease.[24,27] Tumor diameter ([?]80mm) and LDH level at diagnosis (> x ULN) were reported to be factors significantly related to BM involvement by Inagaki et al.[29] Oberlin et al reported fever at diagnosis was significantly associated with the presence of BM metastasis.[27] 
One study analyzed the concordance between BM aspirate (BMA) and BM biopsy (BMB). Among 59 patients with bilateral BMA and 62 patients with bilateral BMB, concordance rates between sides were $97 \%$ and $98 \%$, respectively. BMB was concluded to be more sensitive to detect metastatic disease than BMA as 100\% (4/4) cases of positive BMA were also found to have positive BMB, three positive BMB procedures had negative ipsilateral BMA.[21] In a study including adult patients with various types of sarcoma, discrepancies in findings of metastatic disease between bilateral samples by morphologic evaluation were found in $29 \%$ of $(27 / 94)$ of cases.[40]

\section{Discussion}

To our knowledge, this systematic review is the first to describe the incidence of metastatic BM disease with and without the presence of additional metastatic disease in newly diagnosed ES patients, as well as report the testing characteristics of FDG-PET scans in the diagnosis of metastatic BM disease in this population.

Our results indicate a pooled incidence of BM metastasis of $4.8 \%$ in all patients with newly diagnosed ES and $17.5 \%$ in patients with other clinically or radiographically apparent metastatic disease. Further, $8.8 \%$ of patients with known lung metastasis were reported to have BM but not bone metastasis. FDG-PET scan demonstrates a sensitivity and specificity of $100 \%$ and $96 \%$, and a PPV and NPV of $75 \%$ and $100 \%$ to detect BM metastatic disease compared to BMBA. Given these findings, we have modified our local practice as follows. During initial workup in patients with newly diagnosed ES presenting with clinical or radiographic (including FDG-PET scan) evidence of metastatic disease, we obtain BMBA as this information may provide information relevant to prognosis, response assessment, and understanding potential delays due to slow blood count recovery. In the specific setting of isolated pulmonary metastasis on staging studies obtained prior to BMBA, the incidence of detecting BM metastatic disease appears high enough and the prognostic impact of a positive finding great enough to make this a particularly important population to evaluate by BMBA. On the converse, one may reasonably conclude that detecting BM metastasis in patients with known bone metastasis provides only modest additional prognostic information. Our local practice nevertheless still includes BMBA for such patients to obtain full extent of disease at baseline, which may inform response, toxicity assessment, and prognosis. In patients with no evidence of metastases including no BM uptake on FDG-PET scan, we now omit BMBA as part of routine staging, unless there are clinical concerns (e.g., findings on peripheral complete blood count) or clinical trial requirements. This latter approach is consistent with conclusions drawn by several studies included in this review. [21,24,26,29] While some studies reported an association of BM metastasis disease with primary tumors of the pelvis, [24,27] it is not clear if this finding represents sampling of primary tumor tissue during BMBA procedure. Based upon the available evidence, we do not recommend bone marrow sampling in the setting of a primary pelvic tumor if diagnostic workup reveals no concern for metastatic lesions.

Though rare, we identified 18 reported cases of newly diagnosed ES patients with isolated BM metastasis. $[24,25,27,36,37]$ In eight cases, location of the primary tumor was provided, with $63 \%$ (5/8) reporting location in the appendicular skeleton.[24,25,27] On review of these individual cases, no significant associations to aid in their identification was observed. In 16 of 18 cases, staging workup was reported. All 16 cases included MRI of the primary site, CT scan of the chest as well as bone scan to evaluate the presence/absence of metastatic disease. FDG-PET scans, a relatively newer technology, were not reported during initial workup.

We acknowledge that our approach to omit BMBA at initial staging in patients without other metastasis may miss this very rare subgroup of patients with isolated BM metastasis. However, the available data support a role for using FDG-PET scan as a screening tool for assessment for BM disease. We found no reported cases of false negative findings of BM metastatic disease by use of FDG-PET scan (sensitivity and NPV of $100 \%$ ). Thus, when FDG-PET scan is negative for BM metastasis or other metastasis, omission of BMBA seems reasonable. When FDG-PET scan demonstrates BM metastasis by imaging, the decision to perform a confirmatory BMBA may be based upon the overall clinical context. For example, an FDG-PET scan demonstrating a positive BM signal in the context of widespread bone metastasis would likely be viewed differently from an FDG-PET scan demonstrating only a positive BM signal in a patient with otherwise 
localized disease. Given the major prognostic implications in the latter case, a confirmatory BMBA may be particularly important clinically. Of note, our review found one reported false positive case by FDG-PET scan with negative BMBA. However, this assumes BMBA as the gold standard for diagnosing BM metastasis, but given the possibility of BM involvement being patchy, this finding may represent a false negative BMBA.

The role of BM investigation by either a BMB or BMA or by a unilateral vs bilateral procedure were discussed in several reports. One study concluded that completing only a BMB or a BMA was inadequate as they noted $\mathrm{BM}$ involvement to be focal in several patients resulting in frequent discrepancies between aspirates and biopsies at the various sites explored.[27] A second study analyzing the ability of BMB vs BMA and unilateral vs bilateral approach in detection of BM metastatic disease found BMB and bilateral approach, respectively, were superior in detecting BM metastases.[21] Based upon these findings, when indicated, we obtain bilateral evaluation with bone aspirate and biopsy.

From this systematic review of the literature, the incidence of BM metastasis is $4.8 \%$ in all patients with newly diagnosed ES and $17.5 \%$ in patients with clinically apparent metastatic ES. Based upon the testing characteristics of FDG-PET scans in the diagnosis of metastatic BM disease in newly diagnosed ES, we now obtain BMBA at initial staging according to the algorithm in Figure 2. In the absence of specific extenuating circumstances, we omit staging BMBA in newly diagnosed patients with no evidence of metastases on other staging studies. In patients with newly diagnosed ES who have metastatic disease detected by other staging studies that include an FDG-PET scan, we obtain baseline BMBA.

Conflict of Interest Statement: SGD reports travel expenses from Loxo Oncology, Roche, and Salarius and consulting fee from Bayer and Loxo Oncology.

Grant Support: NIH T32 CA136432-08 (KC) and Alex's Lemonade Stand Foundation (KC \& DS).

\section{REFERENCES}

1. Grunewald TGP, Cidre-Aranaz F, Surdez D, et al. Ewing sarcoma. Nat Rev Dis Primers 2018:4(1):5.

2. Ladenstein R, Potschger U, Le Deley MC, et al. Primary disseminated multifocal Ewing sarcoma: results of the Euro-EWING 99 trial. J Clin Oncol 2010:28(20):3284-3291.

3. Cotterill SJ, Ahrens S, Paulussen M, et al. Prognostic factors in Ewing's tumor of bone: analysis of 975 patients from the European Intergroup Cooperative Ewing's Sarcoma Study Group. J Clin Oncol 2000:18(17):3108-3114.

4. Thiel U, Wawer A, von Luettichau I, et al. Bone marrow involvement identifies a subgroup of advanced Ewing sarcoma patients with fatal outcome irrespective of therapy in contrast to curable patients with multiple bone metastases but unaffected marrow. Oncotarget 2016:7(43):70959-70968.

5. Gaspar N, Hawkins DS, Dirksen U, et al. Ewing Sarcoma: Current Management and Future Approaches Through Collaboration. J Clin Oncol 2015:33(27):3036-3046.

6. Biermann JS, Chow W, Reed DR, et al. NCCN Guidelines Insights: Bone Cancer, Version 2.2017. J Natl Compr Canc Netw 2017:15(2):155-167.

7. Bain BJ. Bone marrow biopsy morbidity: review of 2003. J Clin Pathol 2005:58(4):406-408.

8. Mody RJ, Bui C, Hutchinson RJ, et al. FDG PET imaging of childhood sarcomas. Pediatr Blood Cancer 2010:54(2):222-227.

9. Behzadi AH, Raza SI, Carrino JA, et al. Applications of PET/CT and PET/MR Imaging in Primary Bone Malignancies. PET Clin 2018:13(4):623-634.

10. Guimaraes JB, Rigo L, Lewin F, et al. The importance of PET/CT in the evaluation of patients with Ewing tumors. Radiol Bras 2015:48(3):175-180. 
11. Anderson PM. Futility versus utility of marrow assessment in initial Ewing sarcoma staging workup. Pediatr Blood Cancer 2015:62(1):1-2.

12. Balamuth NJ, Womer RB. Ewing's sarcoma. Lancet Oncol 2010:11(2):184-192.

13. Volker T, Denecke T, Steffen I, et al. Positron emission tomography for staging of pediatric sarcoma patients: results of a prospective multicenter trial. J Clin Oncol 2007:25(34):5435-5441.

14. Kumar J, Seith A, Kumar A, et al. Whole-body MR imaging with the use of parallel imaging for detection of skeletal metastases in pediatric patients with small-cell neoplasms: comparison with skeletal scintigraphy and FDG PET/CT. Pediatr Radiol 2008:38(9):953-962.

15. Kneisl JS, Patt JC, Johnson JC, et al. Is PET useful in detecting occult nonpulmonary metastases in pediatric bone sarcomas? Clin Orthop Relat Res 2006:450:101-104.

16. Gyorke T, Zajic T, Lange A, et al. Impact of FDG PET for staging of Ewing sarcomas and primitive neuroectodermal tumours. Nucl Med Commun 2006:27(1):17-24.

17. Franzius C, Sciuk J, Daldrup-Link HE, et al. FDG-PET for detection of osseous metastases from malignant primary bone tumours: comparison with bone scintigraphy. Eur J Nucl Med 2000:27(9):13051311.

18. Harrison DJ, Parisi MT, Shulkin BL. The Role of (18)F-FDG-PET/CT in Pediatric Sarcoma. Semin Nucl Med 2017:47(3):229-241.

19. Eugene T, Corradini N, Carlier T, et al. (1)(8)F-FDG-PET/CT in initial staging and assessment of early response to chemotherapy of pediatric rhabdomyosarcomas. Nucl Med Commun 2012:33(10):1089-1095.

20. Treglia G, Salsano M, Stefanelli A, et al. Diagnostic accuracy of (1)(8)F-FDG-PET and PET/CT in patients with Ewing sarcoma family tumours: a systematic review and a meta-analysis. Skeletal Radiol 2012:41(3):249-256.

21. Newman EN, Jones RL, Hawkins DS. An evaluation of [F-18]-fluorodeoxy-D-glucose positron emission tomography, bone scan, and bone marrow aspiration/biopsy as staging investigations in Ewing sarcoma. Pediatr Blood Cancer 2013:60(7):1113-1117.

22. Daldrup-Link HE, Franzius C, Link TM, et al. Whole-body MR imaging for detection of bone metastases in children and young adults: comparison with skeletal scintigraphy and FDG PET. AJR Am J Roentgenol 2001:177(1):229-236.

23. Paulussen M, Craft AW, Lewis I, et al. Results of the EICESS-92 Study: two randomized trials of Ewing's sarcoma treatment-cyclophosphamide compared with ifosfamide in standard-risk patients and assessment of benefit of etoposide added to standard treatment in high-risk patients. J Clin Oncol 2008:26(27):4385-4393.

24. Cesari M, Righi A, Colangeli M, et al. Bone marrow biopsy in the initial staging of Ewing sarcoma: Experience from a single institution. Pediatr Blood Cancer 2019:66(6):e27653.

25. Khan SJ, Ishaq I, Saeed H, et al. Bone Marrow Involvement in Metastatic Pediatric Ewing Sarcoma. J Coll Physicians Surg Pak 2017:27(8):502-504.

26. Kopp LM, Hu C, Rozo B, et al. Utility of bone marrow aspiration and biopsy in initial staging of Ewing sarcoma. Pediatr Blood Cancer 2015:62(1):12-15.

27. Oberlin O, Bayle C, Hartmann O, et al. Incidence of bone marrow involvement in Ewing's sarcoma: value of extensive investigation of the bone marrow. Med Pediatr Oncol 1995:24(6):343-346.

28. Wexler LH, DeLaney TF, Tsokos M, et al. Ifosfamide and etoposide plus vincristine, doxorubicin, and cyclophosphamide for newly diagnosed Ewing's sarcoma family of tumors. Cancer 1996:78(4):901-911. 
29. Inagaki $\mathrm{C}$, Shimoi $\mathrm{T}$, Sumiyoshi Okuma H, et al. Bone marrow examination in patients with Ewing sarcoma/peripheral primitive neuroectodermal tumor without metastasis based on (18)F-fluorodeoxyglucose positron emission tomography/computed tomography. Med Oncol 2019:36(7):58.

30. Kasalak O, Glaudemans A, Overbosch J, et al. Can FDG-PET/CT replace blind bone marrow biopsy of the posterior iliac crest in Ewing sarcoma? Skeletal Radiol 2018:47(3):363-367.

31. Albano D, Dondi F, Schumacher RF, et al. Clinical and Prognostic Role of 18F-FDG PET/CT in Pediatric Ewing Sarcoma. J Pediatr Hematol Oncol 2020:42(2):e79-e86.

32. Cangir A, Vietti TJ, Gehan EA, et al. Ewing's sarcoma metastatic at diagnosis. Results and comparisons of two intergroup Ewing's sarcoma studies. Cancer 1990:66(5):887-893.

33. Worch J, Ranft A, DuBois SG, et al. Age dependency of primary tumor sites and metastases in patients with Ewing sarcoma. Pediatr Blood Cancer 2018:65(9):e27251.

34. Vietti TJ, Gehan EA, Nesbit ME, Jr., et al. Multimodal therapy in metastatic Ewing's sarcoma: an Intergroup Study. Natl Cancer Inst Monogr 1981(56):279-284.

35. Oberlin O, Rey A, Desfachelles AS, et al. Impact of high-dose busulfan plus melphalan as consolidation in metastatic Ewing tumors: a study by the Societe Francaise des Cancers de l'Enfant. J Clin Oncol 2006:24(24):3997-4002.

36. Bernstein ML, Devidas M, Lafreniere D, et al. Intensive therapy with growth factor support for patients with Ewing tumor metastatic at diagnosis: Pediatric Oncology Group/Children's Cancer Group Phase II Study 9457-a report from the Children's Oncology Group. J Clin Oncol 2006:24(1):152-159.

37. Miser JS, Goldsby RE, Chen Z, et al. Treatment of metastatic Ewing sarcoma/primitive neuroectodermal tumor of bone: evaluation of increasing the dose intensity of chemotherapy-a report from the Children's Oncology Group. Pediatr Blood Cancer 2007:49(7):894-900.

38. Kasalak O, Dammann A, Adams HJA, et al. Surveillance MRI for the detection of locally recurrent Ewing sarcoma seems futile. Skeletal Radiol 2018:47(11):1517-1522.

39. Yagci-Kupeli B, Kocyigit-Deveci E, Adamhasan F, et al. The Value of 18F-FDG PET/CT in Detecting Bone Marrow Involvement in Childhood Cancers. J Pediatr Hematol Oncol 2019:41(6):438-441.

40. Wang J, Weiss LM, Chang KL, et al. Diagnostic utility of bilateral bone marrow examination: significance of morphologic and ancillary technique study in malignancy. Cancer 2002:94(5):1522-1531.

\section{Figure Legends:}

Figure 1. Selection flow chart for studies included in the systematic review.

Figure 2. Algorithm for bone marrow staging for patients with newly diagnosed Ewing sarcoma based upon data pooled from systematic review.

\section{Hosted file}

Table_1.docx available at https://authorea.com/users/360472/articles/482161-role-of-bonemarrow-biopsy-for-staging-new-patients-with-ewing-sarcoma-a-systematic-review

\section{Hosted file}

Table_2.docx available at https://authorea.com/users/360472/articles/482161-role-of-bonemarrow-biopsy-for-staging-new-patients-with-ewing-sarcoma-a-systematic-review

\section{Hosted file}

Table_3.docx available at https://authorea.com/users/360472/articles/482161-role-of-bonemarrow-biopsy-for-staging-new-patients-with-ewing-sarcoma-a-systematic-review 

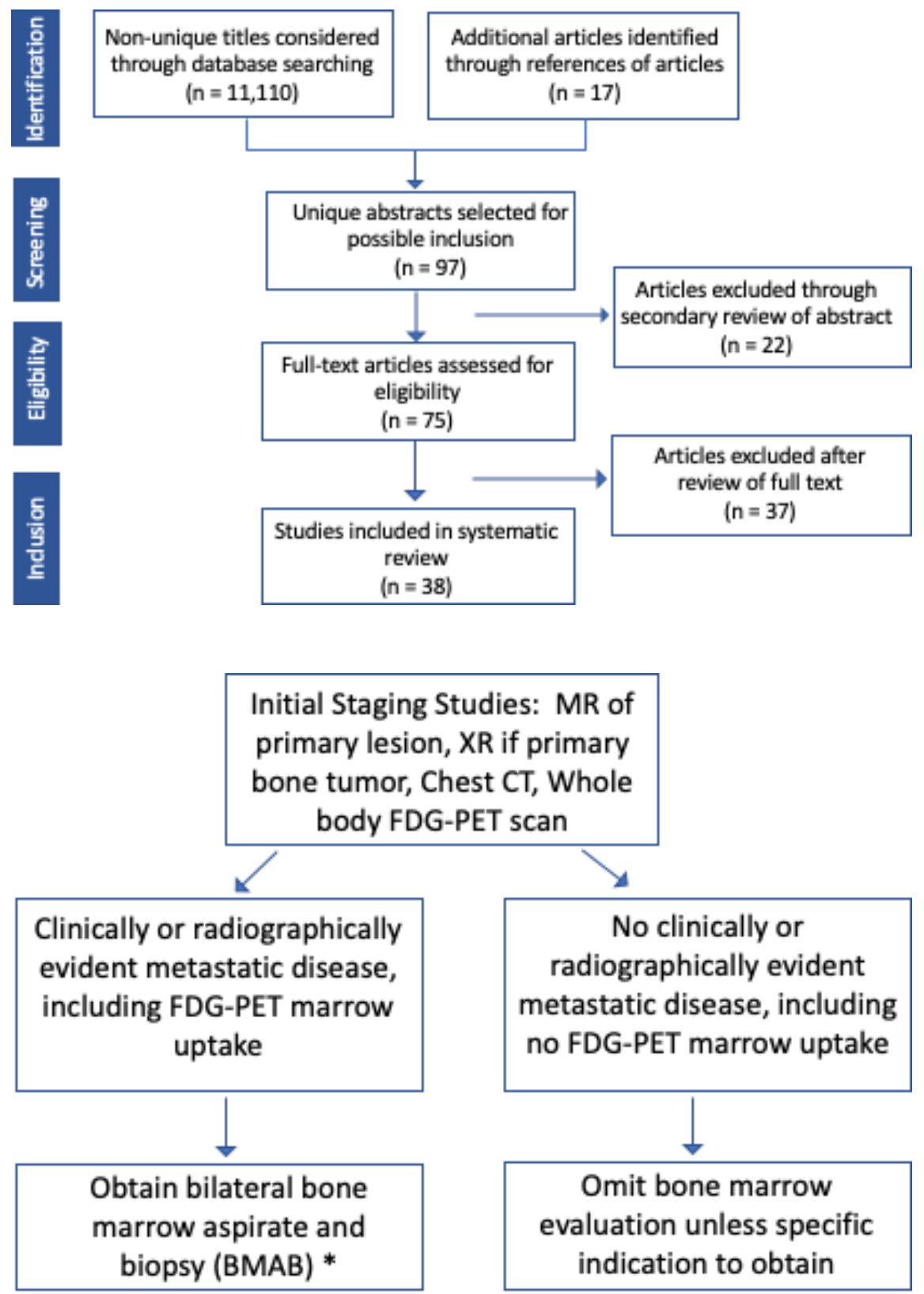

*Decision to obtain BMAB for an individual patient may be influenced by involvement of other metastatic sites since greater prognostic impact in patients with otherwise isolated lung metastasis relative to prognostic impact in patients with bone metastasis. 